\title{
Wachsende Bedeutung des Rechts für die Geographie
}

Eine Thesesoll am Anfang unserer Überlegungen stehen: Je mehr sich die Geographie mit Teilen ihrer Wissenschaft um das Angehen konkreter Probleme bemüht, desto größer wird für sie die Relevanz des Rechts und der Rechtswissenschaft. Die Gegenthese ist gleich zur Hand: Je mehr sich die Geographie auf die Beschreibungsfunktion politisch-wirtschaftlich-sozialer und natürlicher Gegebenheiten zurückzieht und bei der Analyse entsprechender Tatbestände und Prozesse ihre Aufgabe als beendet betrachtet, desto kleiner ist für sie die Bedeutung des Rechts.

Der Anla $\beta$ für die Thesen ist eigentlich banal. Sie drängten sich mir auf, weil ich mich mit dem Phänomen auseinanderzusetzen habe, daß die Geographiestudenten, die ins Nachdiplomstudium für Raumplanung an der ETH Zürich eintreten, bis heute kaum elementare Rechtskenntnisse mitbringen. Nur einige wenige haben im Verlauf ihres Studiums die eine oder andere Rechtsvorlesung besucht, ohne allerdings den Stellenwert des Rechts für ihre Aufgabe voll zu erkennen. Wie sich die Studenten dann aber mit Fragen der Raumplanung, des Natur- und Heimatschutzes, der Bodenpolitik, des Umweltschutzes, der Regionalpolitik, der Regionalwirtschaft usw. befassen sollen, kommen sie nicht mehr darum herum, die Aussagen der Rechtsordnung zur Kenntnis zu nehmen und mit ihnen zu arbeiten. Unvermittelt stehen sie mitten in der Lehre vom Recht und werden von der unendlichen Vielzahl positiver Rechtsnormen arg geschüttelt, bis hin zur «Prüfungsverzweiflung», dennoch begleitet von der wachsenden Einsicht, daß es ohne dieses merkwürdige Gebilde des Rechts nicht geht. Anfänglich wird zwar noch oft und gern der Fluchtweg zur zweiten These, der Gegenthese angetreten, doch spätestens nach zwei, drei Jahren der Praxis schlägt sich der Geograph mit dem "ach so lästigen Recht» herum.

\section{Grundlegendes Bezugsverhältnis}

Wenn die erste These stimmt, dann ist die Folgerung gegeben: Eine minimale Beschäftigung mit dem Recht ist unausweichlich. Allerdings, selbst wenn die zweite These richtig sein sollte, gehört das Recht zum Gegenstand des Interesses der Geographen, ist doch das Recht Teil der kulturellen Wirklichkeit mit Ausstrahlungen in das politische, wirtschaftliche und soziale Geschehen und deshalb notwendiger und wohl auch essentieller Teil der Humanund Wirtschaftsgeographie.' Sicherlich, für die physische
Geographie mit ihrem naturwissenschaftlichen Rückbezug mag das Recht irrelevant sein, doch sind ihre Erkenntnisse letztlich nur dann von Aussagekraft, wenn sie in Korrelation zur gesellschaftlichen Wirklichkeit gestellt und Grundlage für soziales und politisches Entscheiden, allenfalls sogar für Verhaltensänderungen werden, die, rechtlich gestützt, sicherlich nicht gebremst werden sollen.

Auf der andern Seite steht die betont eingebrachte erste These im Vordergrund, ergreift doch die Geographie in steigendem Maße das Wort in Fragen der Raumplanung, des Umweltschutzes und der wirtschaftlichen Strukturpolitik - lokal, regional, national und sogar global - und nimmt sie Einfluß auf die Ausrichtung möglicher Problemlösungen; oft wirkt sie sogar aktiv an der Bewältigung konkreter offener Fragen mit. Die zahlreichen Mitarbeiter mit dem Grundstudium Geographie in Planungsund Umweltschutzbüros des privatwirtschaftlichen Sektors und bei der öffentlichen Hand sprechen für sich selbst. Aber auch in der Wissenschaft, in der Lehre von der Raumplanung und vom Umweltschutz wirken Geographen aktiv mit. Mindestens für die Schweiz darf zudem hinzugefügt werden, daß die Geographen an der Grundlegung der wissenschaftlichen Einbettung der Raumplanung und des Umweltschutzes sowie der Regionalpolitik aktiv beteiligt waren und sind. Die Namen von Heinrich Gutersohn und Ernst Winkler - sie stehen für viele! - sind der Beweis. Beide haben sich aktiv um die rechtliche Grundlegung der «Landesplanung» bemüht, an vorderster Stelle Heinrich Gutersohn, der in den sechziger Jahren die Expertenkommission für die Landesplanung leitete und einen Schlußbericht vorlegte, der weitgehend juristisch geprägt ist. $^{2}$

\section{Neue öffentliche Aufgaben}

Die Nähe zum Recht ist für die Geographie nicht nur deshalb unabdingbar, weil sich die Geographen mit ihrem Berufsfeld näher zur konkreten Problemlösung hinbewegen, sondern auch deshalb, weil das Recht in steigendem $\mathrm{Maße}$ in das gesellschaftiche, wirtschaftiche und politische Leben eingreift. Die Stichworte der Verrechtlichungsten-

Martin Lendi, Prof. Dr., Professor für Rechtswissenschaft, Vorsteher Departement Recht und Ökonomie, ETH Zürich 
denz und der Regulierungsneigung weisen auf das Phänomen hin, doch erklären sie mit ihrem immanent negativ wertenden Urteil die Umstände nur unzulänglich.

In unserem Zusammenhang interessiert vor allem die Tatsache, daß sich das Recht mit neuen öffentlichen Aufgaben, wie Raumplanung, Umweltschutz, Regionalwirtschaft, aber auch Natur-, Landschafts- und Denkmalschutz, sodann mit einer vernetzten Wirklichkeit von Wirtschaft und Staat, resp. Wirtschaft und Gesellschaft auseinandersetzen muß. Den Hintergrund dazu bilden die rasant steigenden Ansprüche an den Lebensraum mit den von ihnen ausgehenden Gefährdungspotentialen. Sie zwangen und zwingen die Politik, die Herausforderung des sorgfältigen Umganges mit den Ressourcen und also unter anderem mit den Aufgäben der Raumplanung und des Umweltschutzes anzunehmen und die Rechtsordnung um diese neuen Aufgabenfelder zu ergänzen. ${ }^{3}$ Und so stehen wir heute vor der Tatsache, daß so wichtige öffentliche Aufgaben wie Raumplanung und Umweltschutz eine positivrechtliche Regelung erfahren haben und also nicht mehr ohne die Vorgaben und Einbindungen des Rechts an die Hand genommen werden können. Raumplanung und Umweltschutz ohne Rückbindung an das Recht betreiben zu wollen, ist damit schlicht und einfach nicht mehr möglich, weil das Recht deren Träger, die Ziele, die Instrumente und die Maßnahmen wie auch die Verfahren festlegt. Selbstredend kommt es immer wieder zu Erkenntnissen, welche die Grenzen des Rechts aufzeigen und die nach Rechtsmodifikation rufen, doch ändert dies nichts daran, daß letztlich nur jene Maßnahmen ergriffen werden können, die vom Recht her vorgesehen und mit Verbindlichkeit ausgerïstet sind, ist doch die Rechtsverbindlichkeit Voraussetzung der Durchsetzbarkeit. An diesen Beispielen der Raumplanung und des Umweltschutzes ist also erkennbar, daß die wachsende Bedeutung des Rechts nicht mit negativen Schlagworten abgetan werden kann, sondern eine notwendige Antwort auf konkrete, neuartige Problemlagen ist, die angenommen werden müssen. Auf der andern Seite bleibt unbestritten, daß des Politikers schneller Weg zum Gesetz nicht immer die beste Lösung ist und daß deshalb Überregulierungen abgebaut werden müssen.

\section{Der Internationalisierungsschub}

Völlig unabhängig von unserer positiven oder kritischen Einstellung zum Recht ist sodann eine zweite Kraft auszumachen, die faktisch zu einem Bedeutungszuwachs für das Recht führt. Es ist dies die internationale Rechtsentwicklung. War es bis vor wenigen Jahren durchaus zureichend, sich im nationalen Recht einigermaßen auszukennen und die wichtigsten Gesetze handhaben zu können, so stehen wir heute vor der Tatsache, daß das nationale Rechtsgebäude in das internationale verwoben ist. Das heutige Völkerrecht beschränkt sich nicht mehr auf Aussagen zu zwischenstaatlichen Konfliktfeldern wie Krieg und Frieden oder zu Fragen der Nachbarschaft - grenzüberschreitende Probleme -, sondern behandelt vor dem
Hintergrund der Globalisierung aller wichtigen Problemfelder faktisch die gleiche Traktandenliste wie das nationale Recht. ${ }^{4}$ Es gibt eben heute nicht nur ein kommunales, kantonales und nationales Raumplanungs- und Umweltschutzrecht, sondern auch ein internationales, wie auch das Wasserwirtschaftsrecht längst über die nationale Ebene hinausgewachsen ist, vom Verkehrsrecht mit seinem Telekommunikations- und dem Luftfahrtbereich nicht zu reden.

Besonders eindrücklich ist die Rechtsentwicklung im $\mathrm{Zu}$ sammenhang der Europäischen Gemeinschaften, deren Verordnungen und Richtlinien nach schweizerischem Rechtsverständnis, sollten wir der EG oder dem EWR beitreten, dem nationalen Recht vorgehen, und das in hohem Maße nicht nur einige klassische Belange der internationalen Kooperation beschlägt, sondern alle wichtigen Teile des Wirtschaftsrechts, aber auch der Umweltund der Regionalpolitik und bald auch einmal der AuBen- und Sicherheitspolitik betrifft. ${ }^{5}$ Die großen Umwelt konferenzen von Stockholm und Rio de Janeiro haben zwar nur begrenzt zum Erlaß von "hartem» Völkerrecht geführt, doch ist das "soft law», das in Resolutionen und Empfehlungen seinen Ausdruck findet, nicht einfach wegzudisputieren, da es die Staaten, die ihm zugestimmt haben, mindestens politisch bindet und weil es sich früher oder später zum Kernbestand des Völkerrechts verdichten kann.

Die «Interaktion» zwischen internationalem und nationalem Recht wird nachvollziehbar am konkreten Beispiel der Landwirtschafispolitik. Genügte es bis vor kurzem, einen Blick auf die Landwirtschaftsgesetzgebung des Bundes zu werfen, so ist es heute unabdingbar, das internationale Recht mitzunehmen, konkret: das GATT-Recht und das EG-Recht, ${ }^{6}$ wird sich doch die Weiterentwicklung des schweizerischen Landwirtschaftsrechts von dort her bestimmen. Selbst vermeintlich nationale Verkehrsprobleme haben stets einen internationalen Hintergrund, da die Mobilität längst internationale Dimensionen angenommen hat und vor nationalen Grenzen nicht mehr halt zu machen gewillt ist. Auch wenn die EG relativ spät mit der Forcierung des Verkehrsrechts eingesetzt hat, so muß heute akzeptiert werden, daß das internationale Verkehrsrecht bestimmend Einfluß nimmt, sogar mit Vernetzungsanliegen in Richtung Umweltschutz und Energiebelange. Ähnliches wäre über das Recht der Entwicklungspolitik zu berichten.

Der Jurist spürt die Internationalisierung des Rechts bis in den Alltag hinein. Handel und Gewerbe überspringen die Grenzen von Tag zu Tag: Zukauf und Verkauf von Firmen an international tätige Firmen gehören zum Alltag, Kaufverträge betreffen längst unendlich viele Geschäfte innerhalb und außerhalb Europas. Das Recht hat darauf reagiert: Das Kaufvertragsrecht wird internationalisiert, und das Prozeßrecht muß die internationalen Bezüge aufnehmen. Entscheidend aber ist, daß die Entwicklungskräfte erkannt werden. Diese weisen ganz allgemein auf die Internationalisierung des Rechts hin, mit Wirkung für die meisten Bereiche menschlicher Aktivitäten und Verantwortlichkeiten. 


\section{Komplexe Wirklichkeit - vernetztes Recht}

Mehr Rechtssätze wegen neuer öffentlicher Aufgaben, eine zusätzliche Rechtserzeugungsebene durch die Internationalisierung des Rechts - beide Sachverhalte machen den Umgang mit dem Recht nicht einfacher. Ein drittes Element ist dabei zusätzlich zu beachten: Die Sachverhalte, denen die "Problemlöser» auf der Grundlage des Rechts zu begegnen haben, sind nicht einfacher, sondern wesentlich schwieriger zu handhaben geworden. Bedarf es der Beispiele?

Ist eine Deponie zu errichten, so sind damit sachliche Aspekte der Abfallentsorgung verbunden, dann aber auch Probleme der Raumplanung, und zwar auf überörtlicher und örtlicher Ebene, sodann Fragen des Baurechts, des Gewässerschutzrechts und vor allem auch solche des Umweltrechts - bis hin zum Verkehrsrecht und zum Forstpolizeirecht, falls mit einer Deponie erheblicher Verkehr verbunden sein oder eine Rodung nötig werden sollte.? Daraus resultiert die Notwendigkeit der gleichzeitigen Anwendung mehrerer Gesetze und die Durchführung mehrerer Verfahren auf den einen Tatbestand der Errichtung einer konkreten Deponie; ein äuBerst schwieriger Vorgang, der mit der ueinfachen Rechtsanwendung», die von einem einzigen Rechtssatz ausgeht, kaum mehr etwas zu tun hat. Die Rechtsanwendung zur Meisterung täglicher Probleme ist also äußerst kompliziert geworden! Zwei Stichworte zeigen dies an: Koordinierte Anwendung des materiellen Rechts, beispielsweise des Umwelt-, Gewässerschutz-, Raumplanungsund Baurechts, sowie Koordination der Verfahren bei unterschiedlichen Zuständigkeiten - Nutzungsplanungs-, Baubewilligungs-, Deponie- und Gewässerschutzbewilligungs- sowie Rodungsbewilligungsverfahren. Dabei wird sich gleich auch einmal bemerkbar machen, daß es nicht mehr genügt, zum Gesetz zu greifen. Vielmehr wird zusätzlich die Rechtsprechung zu konsultieren sein, die Hinweise gibt, wie solche Probleme gemeistert werden können.

Ähnliche Überlegungen könnten am Beispiel der Führung einer Starkstromleitung durch eine schützenswerte Landschaft oder an der baulichen Verwirklichung der Bahn 2000 oder sogar der NEAT entwickelt werden. ${ }^{8}$ Die Errichtung eines Flughafens war im Zeitpunkt der Planung und des Baus des Flugplatzes Kloten vergleichsweise noch einfach zu handhaben, doch dürfte ein weiterer Ausbau heute oder morgen von vornherein in rechtliche Probleme hineinführen, die mit der Anwendung des Luftfahrtgesetzes allein nicht bewältigt werden können. Der Grund liegt dabei weniger bei der an Dickenwachstum leidenden Rechtsordnung, sondern primär bei der komplexen Wirklichkeit, also beim allseits verwobenen Sachverhalt, der die sektoral konzipierte Gesetzgebung herausfordert, und dies erst noch auf allen Ebenen: von der Gemeinde bis zum Recht der EG. Selbst scheinbar örtliche Probleme, wie die Bauwerks- und Siedlungserneuerung, geprägt durch die Zielsetzung der besseren Nutzung des Bestehenden, rufen einer eleganten Rechtsanwendung einer Vielzahl von Rechtsnormen, ist doch al- lein schon das verdichtete Bauen eine Herausforderung für das Planungs- und Baurecht, ${ }^{9}$ wie auch für das Umweltschutz- und das Denkmalschutzrecht, mit weiteren Hinweisen auf die Gestaltungsbelange und die Erschließung. Wie dem auch sei, ohne Übersicht über die Rechtsordnung und ohne die Fähigkeit, in sie einzudringen, ist vor diesem Hintergrund wohl kaum mehr Problemlösungskompetenz zu erlangen.

Und noch ein weiteres Beispiel? Allein schon die Begegnung von Umweltschutz- und Raumplanungsrecht wirft viele Fragen auf, die ohne die Interpretationsbegabung, wie das Umweltrecht und wie das Raumplanungsrecht zu verstehen sind, nicht mehr angegangen werden können. In welchem Maße kann und darf vom Umweltschutzrecht her auf die Nutzung von Zonen und von Erschließungsanlagen sowie von einzelnen Bauten Einfluß genommen werden? Wer ist zuständig, die räumlichen Vorstellungen, resultierend aus und für immissionsvorbelastete(n) Räume(n), einzubringen - die Raumplanung oder der Umweltschutz? So besehen dürfte nachvollziehbar geworden sein, wie bedeutsam die Fähigkeit im Umgang mit dem Recht für all diejenigen geworden ist, die aktuelle Probleme angehen wollen oder müssen.

\section{Verständliche Hemmschwellen}

Vor dieser Sachlage, die zum Recht drängt, müßte es an sich für alle Problemlöser selbstverständlich sein, den Schritt zum Recht zu wagen. Leider ist dem nicht so. Manche Ökonomen, viele Geographen und sogar einige Politologen wie auch Politik-Philosophen schrecken vor dem Recht zurück. Der Grund ist zunächst einfach auszumachen. Wer von der Mittelschule her kommt, der hat kaum je Rechtsunterricht genossen, sieht man von der Rechtskunde in den Handelsfächern ab. Der üblicherweise angebotene Staatskunde-Unterricht bewegt sich mehr oder weniger auf der Ebene der Kenntnisse der Staatsfunktionen, der Entscheidungsprozesse und der Ansprechbarkeit der Institutionen, allenfalls ausgeweitet in die Diskussion erlebbarer Politik mit Brücken zum Parteigeschehen, zur Bedeutung der Verbände, möglicherweise auch mit Verständnisbemühungen um die verfassungsrechtliche Grundorientierung gegenüber dem Individuum, der Wirtschaft und der Gesellschaft, ergänzt durch praktische Beispiele der Sachpolitiken, wie Energie, Verkehr oder Siedlung. Dabei gelingt es selten oder nie, das Recht als Recht anzusprechen und in ihm und mit ihm zu denken. Dies ist auch nicht einfach, da das Recht nach normativem Denken verlangt, das in den traditionellen Fächern der Mittelschule nicht vorkommt, bewegt sich doch der gängige Unterricht jenseits der Frage, was man problemlösend tun soll resp. sogar muß. Und so gibt es keinen leichten Einstieg in das Recht, weder für den Mittelschüler noch für den über die Matura ins Studium vorgestoßenen Studenten. Er weicht deshalb vor einem Fach, das im Gymnasium nicht unterrichtet wurde, in- 
stinktiv zurück, resp. er erahnt, daß er nur mit einem "geistigen Ruck» in das ihm fremde Recht eindringen könnte. Damit wären wohl einige Mühen verbunden.

Eine weitere Hemmschwelle liegt in den tieferen Gründen der Verbindlichkeit des Rechts, eine geheimnisvolle Aussage, aber doch eine zentrale. Wer aufbricht, um die Welt zu entdecken und sie allenfalls auch zu verändern, der scheucht vor einer Disziplin, die Verbindlichkeit beansprucht, durch die man in Verantwortung genommen wird, zurück, vor allem, wenn er beiläufig noch erfährt, daß das Recht mit seinem Anspruch auf Geltung moralische Qualitäten und also Wertungen verbindet, von denen man gerade frei sein möchte, um unvoreingenommen an die Probleme herantreten zu können. So besehen ist es nachvollziehbar, wenn vor allem Studenten der Naturwissenschaften das Recht und den Rechtsunterricht als überflüssig betrachten. Auch dem Sozialwissenschaftler, soweit er dem beobachtenden und analysierenden Feststellen und Beschreiben zugetan ist, mag das Recht nicht wichtig erscheinen, selbst wenn er zuzugeben bereit ist, daß es als kulturelles Phänomen nicht ohne Einfluß auf soziale Tatbestände ist und folglich mitgenommen werden müßte. Der Schritt in Richtung auf das Angehen von Problemen, bei dem das Recht zunehmend von Bedeutung wird, der wird auch auf der Stufe des akademischen Studiums nur in einzelnen Disziplinen gemacht - und erst noch relativ spät in höheren Semestern -, mit dem Ergebnis, daß für den Einbezug des Rechts kaum mehr hinreichend Zeit bleibt. So ist es nachvollziehbar, daß auf den Einstieg in die Rechtswissenschaft und in das Recht selbst auf Hochschulebene gern und oft verzichtet wird - leider.

\section{Praktische Hilfestellungen}

Sind die Hemmschwellen zum Recht einmal überwunden, dann blitzen bald seine Hilfen auf.

Befaßt sich beispielsweise ein planender Problemlöser mit der Dimensionierung von Bauzonen, dann erfährt er vom Gesetz, wer für die Zonenplanung zuständig ist, in welchem Verfahren Pläne festgelegt resp. geändert werden, ob und in welcher Art und Weise auf übergeordnete Pläne Rücksicht zu nehmen ist. ${ }^{10} \mathrm{Er}$ findet im geltenden Recht sogar gesetzliche Vorgaben für die Bemessung der Bauzonen, indem dieses umschreibt, welches Land den Bauzonen zuzuscheiden ist: Land, das sich für die Überbauung eignet und das a) weitgehend überbaut oder b) voraussichtlich innert 15 Jahren benötigt und erschlossen wird. " Greift er zusätzlich auf die Rechtsprechung zurück, so kann er dieser entnehmen, daß das Bundesgericht in konstanten Formulierungen davon ausgeht, da $\beta$ die richtige Dimensionierung der Bauzonen im öffentlichen Interesse liegt und daß deshalb gebotene Redimensionierungen vorzunehmen sind - ebenfalls im öffentlichen Interesse. ${ }^{12}$ Kurzum, der Problemlöser erfährt, unter welchen "Randbedingungen» er agieren kann und daß er gewisse Überlegungen nicht ab ovo durchzufüh- ren hat, weil der Gesetzgeber gewisse Festlegungen bereits getroffen hat.

Taucht der Praktiker noch tiefer in das Recht ein, so erfährt er einiges über die Möglichkeiten und Grenzen des Umganges mit den vom Geschehen Berührten. Bleiben wir beim Beispiel der Dimensionierung von Bauzonen mit allfälligen Rückzonungen --, so kann er dem Recht entnehmen, ob und wie die Planungsbetroffenen in den Planungsproze $\beta$ einzubeziehen sind, welche demokratisch gewählten Behörden über die Planfestsetzung entscheiden, welche Rechtsmittel die von der Planung Berührten ergreifen können und in welchem Verfahren der Plan die Abstimmung mit übergeordneten Plänen erlangt und abschließend genehmigt wird, kurzum, er findet eine definierte Gruppe von Planungsbetroffenen, Planungsberührten und Planungsbeteiligten vor, mit denen er sich problemlösend auseinandersetzen soll. ${ }^{13}$

Gehen wir noch einen Schritt weiter, so möchte der Problemlöser wohl wissen, ob seine Dimensionierung der Bauzonen dort, wo es zu sogenannten Rückzonungen kommt, Entschädigungen auslöst, d.h., ob das Gemeinwesen, für das er plant, entschädigungspflichtig wird oder nicht. Es geht hier um das Problem des Schadenersatzes bei rechtmäßigen Handlungen, in concreto: um die sogenannte materielle Enteignungsentschädigung. ${ }^{14}$ Da das Gesetz und die Verfassung lediglich den Grundsatz der vollen Entschädigungspflicht im Falle des Tatbestandes einer materiellen Enteignung festlegen, ist der Planer angewiesen, die Rechtsprechung zu konsultieren, welche die Voraussetzungen näher umschreibt. Aus ihr kann er ableiten, daß das Bundesgericht eine zurückhaltende, aber berechenbare Praxis entwickelt hat, auf deren Grundlage Planungen vorangetrieben werden können. Ein ähnliches Beispiel drängt sich aus dem Gebiet des Umweltschutzes auf. Viele praktisch tätige Geographen sind mit der Aufgabe der Umweltverträglichkeitsprïfung konfrontiert. Um ihre rechtlich relevante Funktion zu verstehen, genügt es nicht, allgemeine Überlegungen zur Umwelt und zum Umweltschutz aus Anlaß eines die Umwelt potentiell belastenden Vorhabens anzustellen. Vorweg gilt es zu bedenken, daß das geltende Recht dieser Umweltverträglichkeitsprüfung materielle und formelle Vorgaben mit auf den Weg gibt. ${ }^{15}$

So ist es nicht unerheblich zu wissen, dass es sich bei der Umweltverträglichkeitsprüfung nicht um ein selbständiges Verfahren, sondern um ein unselbständiges handelt, das einem Bewilligungs- oder Konzessionierungsverfahren beigegeben ist. Im Kern geht es um ein Sonderverfah$r e n$, in dem abgeklärt wird, ob das Projekt den bundesrechtlichen Vorschriften über den Schutz der Umwelt entspricht. Die abschließende Würdigung des Ergebnisses der Umweltverträglichkeitsprüfung steht jenen Behörden zu, die über das Vorhaben zu entscheiden haben, wobei diese letztlich unter der Verantwortung der Anwendung aller Rechtsnormen und nicht ausschließlich jener über den Umweltschutz stehen. Es kann deshalb sein, daß eine Behörde - gestützt auf weitere Gesetzesbestimmungen - gegen einen Umweltverträglichkeits-Be- 
richt entscheiden muß, auch wenn dieser in sich sachlich sorgfältig und rechtlich einwandfrei angelegt ist. Heikle Fragen wirft sodann das Problemfeld des sogenannten maßgeblichen Verfahrens ${ }^{16}$ auf, d. h. jenes Verfahrens, dem das Sonderverfahren der Umweltverträglichkeitsprüfung beigefügt ist, soweit dieses durch die Gesetzgebung nicht expressis verbis bestimmt ist oder auf einen komplexen Sachverhalt mehrerer Verfahren Anwendung findet. Im Sinne der Koordination der Verfahren dürfte das für die Umweltverträglichkeitsprüfung maßgebliche Verfahren dasjenige sein, das die Funktion eines Leitverfahrens zu übernehmen fähig ist, wobei unter mehreren jenes vorzuziehen sein wird, das breitestmögliche Beurteilung in der Sache, frühstmöglichen Einbezug der Planungsbetroffenen, demokratische Mitentscheidung und umfassenden Rechtsschutz erlaubt - eine delikate Frage, die letztlich nur mit Rechtskenntnissen und unter Konsultation der Rechtsprechung zweckmäßig beantwortet werden kann. Nicht minder wichtig ist zu beachten, dass der Prüfungsgegenstand auf die Übereinstimmung mit dem geltenden Umweltschutzrecht ausgerichtet ist, so daß die Durchführung einer Umweltverträglichkeitsprüfung die Kenntnis des positiven Umweltschutzrechts voraussetzt, wobei bereits dessen Umfang eine Frage der Auslegung ist, die durch die Rechtsprechung zu klären ist und auch geklärt wurde, nämlich im Sinne einer nicht abschließenden Aufzählung der in der Verordnung zur Umweltverträglichkeitsprüfung erwähnten Gebiete. ${ }^{17}$ Könnten alle diese Aussagen nicht abgerufen werden, so wäre der Planer resp. Problemlöser wohl eher arg hilflos. Das Recht ist deshalb nicht einfach ein "kompliziertes, weltfremdes» Gebilde, sondern vorweg und vor allem eine Hilfe zur konkreten Problembewältigung. Selbstverständlich kann sich der praktisch tätige Geograph mit einem Juristen besprechen, doch muß er fähig sein, dieses Gespräch zu führen, d.h., er muß in der Lage sein, die rechtserheblichen Fragen aufzuwerfen, was einige Grundlagenkenntnisse voraussetzt.

\section{Der Schritt zum besseren Recht durch gekonnte Rechtsanwendung}

Unter den Hemmschwellen haben wir die Vorurteile gegenüber dem Recht nicht besonders unterstrichen. Sie sind allgemein bekannt. Traditionellerweise wird auf die Starrheit, die Weltfremdheit, die Praxisferne und vor allem auf die Unfähigkeit hingewiesen, im entscheidenden Moment flexible Lösungen einzubringen. Daran mag das eine oder andere richtig sein.

$\mathrm{Zu}$ oft wird von den Kritikern vergessen, daß Gesetze änderbar sind. Auch Pläne sind es. Die Bundesverfassung erklärt sogar, daß sie "jederzeit» geändert werden können - ganz oder teilweise. Und dies sagt eine Verfassung, von der wir lernen, daß sie der Fels im Rechtsgeschehen sein müsse. Zweifellos, die Änderbarkeit des Rechts findet ihre Schranke am Erfordernis der Rechtssicherheit, doch ändert dies nichts daran, daß das Recht reformierbar ist. Erweist sich, daß Recht mangelhaft ist, so muß der Weg zum Gesetzgeber gewagt werden. An ihm ist es, Rechtsänderungen einzuleiten. Es kann der theoretische Satz gewagt werden: Eine allfällig festzustellende erschwerte Änderbarkeit des Rechts ist nicht auf Hindernisse im Recht und durch das Recht zurückzuführen, sondern auf solche in der Politik. Selbstredend kann die Rechtswissenschaft die Verantwortung nicht von sich weisen, zumal sie ihrerseits - aufgrund ihrer Argumentationskraft - rechtspolitische Vorstöße initiieren kann. Unterhalb des Überganges zur formellen Rechtsänderung gibt es nun allerdings zahlreiche Möglichkeiten, vom geltenden Recht her eine den Umständen entsprechende flexible Rechtsanwendungspraxis zu entwickeln. Vorweg ist das Recht durch seine Abstraktion ganz allgemein in der Lage, auf recht unterschiedliche Sachverhalte zu antworten. Dadurch wird jeder Rechtsanwendungsakt zu einem schöpferischen Vorgang. Die Rechtssätze enthalten ferner sogenannte unbestimmte Rechtsbegriffe "wichtiger Grund", "schützenswerte Landschaften", «sanierungsbedürftige Anlage» usw. - , die einen erheblichen Beurteilungsspielraum eröffnen. Außerdem finden sich im positiven Recht Ermessensvorschriften und sogar Ausnahmebewilligungen - alles Instrumente, um die erwartete Flexibilität bei größtmöglicher Rechtssicherheit einzubringen. Voraussetzung ist allerdings, daß man den Umgang mit solchen "Instrumenten» beherrscht, was bereits Methodenkenntnisse bedingt, wie sie aus Urteilen in ähnlichen oder gerade anders gelagerten Fällen hervorgehen.

Wer also als Problemlöser agiert, der kommt nicht nur um das geltende Recht nicht herum, sondern er hat auch Fähigkeiten der kreativen Rechtsanwendung einzubringen. Ohne solche, aus dem Innern des Rechts hervorgehende "Freiheiten» neigt der Nicht-Jurist zum Gesetzesanwendungsautomaten, den das Recht aber nicht will. Ihm ist es ein Anliegen, Beweglichkeit und Rechtssicherheit zu verbinden. Die Auseinandersetzung mit dem Recht fiihrt deshalb nicht in eine ausgrenzende Vertiefung, in einengende Verästelungen, sondern zu einem souveränen Umgang mit dem geltenden Recht - in steter Ausrichtung auf den Sinn einer Vorschrift in ihrem größeren Zusammenhang.

Ein besonders schönes Beispiel geben die Planungsgrundsätze ${ }^{18}$ die der Raumplanung eine gewichtige Ausrichtung vermitteln, ohne den Planer in der Durchführung zu bevormunden. Sie sind anzuwenden, aber in ihrer Offenheit und Widersprüchlichkeit vor ihrer Anwendung untereinander und gegeneinander abzuwägen. $\mathrm{Zu}$ verstehen sind sie letztlich nur von der Tatsache her, daß das Raumplanungsrecht mit seiner Zukunftsdimension relativ offen konzipiert ist. Im wesentlichen enthält das Bundesgesetz über die Raumplanung formelle Vorschriften und vertraut die materiellen Aspekte, abgesehen von einigen sehr zentralen Grundaussagen über die Trennung von Siedlungs- und Nichtsiedlungsgebieten, die Erschließung und das Bauen außerhalb der Bauzonen, diesen «finalen» Rechtssätzen an, die Ziele ansprechen und Handlungsoptionen eröffnen. 


\section{Das Faszinosum «Recht»}

Abgesehen von der Nützlichkeit des Umganges mit dem Recht ist dieses eine faszinierende Größe: Eine von Menschen für Menschen geschaffene verbindliche Ordnung, getragen vom Medium der Sprache, unzulänglich, unvollkommen und doch hervorgegangen mit dem Anspruch, das Zusammenleben der Menschen untereinander und im Einklang mit dem Lebensraum und den Lebensvoraussetzungen verbindlich zu regeln, auf $d a \beta$ das Leben allseits verträglich gelebt werde. ${ }^{19}$

Das etwas altmodisch anmutende, aber bestimmende Stichwort ist dasjenige von der "Friedensordnung». Gegenstand dieser Friedensordnung ist das Zusammenleben der Menschen unter sich und - eine wesentlich jüngere Akzentsetzung - mit der Natur und mit der Umwelt, theologisch betrachtet mit der Schöpfung. Sie ist betont mehrdimensional, da sie, was zu unterstreichen ist, die Menschen, den Lebensraum, die Lebensvoraussetzungen und die Zeit betrifft. Die oft angesprochene "Gerechtigkeit» als innere Rechtfertigung des Rechts kommt erst an nachgeordneter Stelle, zumal die Gerechtigkeitsfrage den Teilaspekt und den Kampf um das Verteilen der Güter einschließt, der nur vertretbar ist, soweit die Friedensordnung vorgegeben ist. Die Rechtsordnung erfüllt weitgehend - ihre Aufgabe, wenn sie die Menschen zu einem fairen Verhalten motivieren und anhalten kann. Nicht zuletzt aus diesem Grund sind Vorschriften über Verfahren, sogenanntes formelles Recht, so enorm wichtig. Sie dürfen nie und nimmer als "formelles Zeug» abgetan werden, zumal sie den fairen Prozeß, die friedliche Auseinandersetzung zum Ziel haben, nicht die Form um der Form willen. So besehen ist das Recht eine kulturelle Leistung, Ausdruck der Kultur menschlicher Hervorhebung geschuldeter Verantwortung für die Menschen und die Natur: Grund genug, ihm Aufmerksamkeit zu schenken.

So wichtig dieser Ansatz ist, hier darf nicht stehengeblieben werden. Zum Faszinosum «Recht» gehört ferner seine Einbindung in die Philosophie, der Rückbezug zur Theologie und - gleichzeitig - die Auseinandersetzung mit der sozialen und natürlichen Wirklichkeit. Gerade weil das Recht bipolar theologisch-philosophische und wirklichkeitsbezogene Relationen einschließt, gerade weil es ethische Urteile mit seinen Aussagen darüber, was getan werden soll, mitnimmt - ohne sich aber zu weit von dem zu entfernen, was durchsetzbar ist -, bleibt das Recht in Bewegung. Die Rechtsordnung lebt, sei es durch schöpferische Rechtsanwendung, sei es durch Rechtsetzung, letztlich von ihren vielschichtigen Dimensionen.

\section{Das Europäische Recht als Novum}

Ein nicht unwichtiger Impuls - um nur einen zu nennen geht vom Europäischen Recht aus. Dabei steht nicht einseitig das EG-Recht im Vordergrund. An dieser Stelle ist vorweg die Europäische Menschenrechtskonvention
(EMRK) zu erwähnen, ${ }^{20}$ die dem verfassungsrechtlichen Denken in allen angeschlossenen Staaten neue Horizonte gegeben und gleichzeitig das eigene geltende Verfassungsrecht zu hinterfragen erlaubte. Der europäische Gerichtshof für Menschenrechte spricht bereits heute Urteile, die schweizerische Entscheidungen in Frage stellen, und das Bundesgericht wendet die Europäische Menschenrechts-Konvention unter Anerkennung von Verfassungsrang an.

Auf die Schweiz kommt sodann das Recht der EG zu,, ${ }^{21}$ sei es aufgrund des Abkommens über den Europäischen Wirtschaftsraum oder aufgrund eines Beitritts zur EG, wie er vom Bundesrat in Aussicht genommen und im Sinne der Einleitung eines abklärenden Beitrittsgesuchverfahrens am 26. Mai 1992 beantragt worden ist. Selbst wenn die Schweiz weder das EWR-Abkommen unterzeichnen noch den Beitritt zur EG beschließen sollte, das EG-Recht wird die schweizerische Rechtsentwicklung nachhaltig beeinflussen. ${ }^{22}$

Im Grunde genommen hat dieser Prozeß bereits begonnen. Unser Land bemüht sich - richtigerweise -, eine mit dem EG-Recht sich auseinandersetzende Rechtsetzung zu betreiben, also Recht zu erlassen, das, wenn nicht dekkungsgleich, so doch kompatibel mit dem EG-Recht ist oder sich willentlich von ihm unterscheidet. Dabei ist es keineswegs so, daß die EG das «bessere» Recht gepachtet hätte, auch wenn nicht zu übersehen ist, daß das $E G$ Recht neue Akzente setzt, die das schweizerische Recht aus Gründen politischer Zurückhaltung nicht anzusprechen wagte. Das heutige EG-Recht des Binnenmarktes ist von seiner ganzen Struktur her, bei aller Weitschweifigkeit in gewissen Richtlinien, tendenzmäßig auf eine freie Handhabung gerichtet, indem es Regulierungen sucht, die Hemmnisse und Diskriminierungen abbauen. Es wird allerdings spannend sein, zu beobachten, ob und wie weit die EG und dereinst die Europäische Union - gemäß den Verträgen von Maastricht vom 7. Februar 1992 - in der Lage ist, diesen Kurs durchzuhalten. Die zunehmende Notwendigkeit, Sozial-, Umwelt-, Energie-, Technologie-, Kohäsions-, Verkehrspolitik usw. betreiben zu müssen, könnte die EG resp. die Europäische Union früher oder später verleiten, Regulierungen nach herkömmlichen Mustern zu erlassen. Wie dem auch sei, die Rechtsordnung der Schweiz erweitert sich um die internationale Dimension in raschen Schritten, wobei es zu verstehen gilt, wie dieses Recht entsteht, wie es sich zum schweizerischen Recht verhält, ob und wie weit es unmittelbar anwendbar ist, wer die Vereinbarkeit von schweizerischem und internationalem Recht zu prüfen hat und wer Interpretationsfragen entscheidet.

\section{Gespräch zwischen Rechtswissenschaft und Geographie}

Es wäre ein Mißverständnis, wenn der Eindruck entstünde, die Rechtswissenschaft möchte sich gleichsam der Geographie aufdrängen. Hingegen müßte die unausweichliche Begegnung der Disziplinen bei der Problem- 
lösung für beide Wissenschaften Anlaß sein, das Gespräch aufzunehmen und von den gegenseitigen Wechselbezügen zu handeln. In diesem Artikel habe ich versucht, von der Seite der Rechtswissenschaft Brücken zu schlagen. Die konkreten Schritte, die sich aus einem Gespräch ergeben könnten, dürften sich vorweg im Unterricht niederschlagen. Auf dieser Ebene werden die Weichen in die Zukunft gestellt.

\section{Anmerkungen}

' Zum Wesen des Rechts vgl. LENDI Martin, Rechtsordnung, Eine Einführung in das schweizerische Recht, mit Tafeln und Beispielen, Zürich 1992 (es handelt sich um ein Lehrmittel für den Rechtsunterricht, verfaßt für Nicht-Juristen).

${ }^{2}$ Bericht der Eidgenössischen Expertenkommission für Fragen der Landesplanung vom 6. Oktober 1966, herausgegeben vom Departement des Innern, Bern 1967.

${ }^{3}$ Art. $22^{\text {quater }}$ BV über die Raumplanung; Art. 24 ${ }^{\text {septies }}$ BV über den Umweltschutz.

${ }^{4}$ Vgl. dazu die Systematische Sammlung des Bundesrechts, die in inrem zweiten Teil das für die Schweiz maßgebende Völkerrecht nach derselben Systematik sammelt wie das nationale Recht.

${ }^{5}$ Der im Vordergrund stehende Vertrag zur Gründung der Europäischen Wirtschaftsgemeinschaft (EWG) vom 25. März 1957 mit seitherigen Änderungen, insbesondere mit Einschluß der "Einheitlichen Europäischen Akte", in Kraft seit 1. Juli 1987, umfaßt neben den auf den Binnenmarkt direkt bezogenen Vorschriften auch solche über den Verkehr, die Umwelt, die Sozialpolitik, Forschung und technologische Entwicklung usw. Die Bedeutung der flankierenden Politiken wächst in Zukunft. Vgl. dazu die Beschlüsse von Maastricht (Fußnote 22).

${ }^{6}$ Allgemeines Zoll- und Handelsabkommen GATT vom 30. Oktober 1947, SR 0.632.21. Zum EG-Recht vgl. die Botschaft des Bundesrates zum EWR-Abkommen vom 20. Mai 1992 sowie den Bericht zur Frage eines Beitritts der Schweiz zur Europäischen Gemeinschaft vom 20. Mai 1992.

${ }^{7}$ Vgl. zur Problematik der rechtlichen Seite der Planung und Errichtung einer Deponie den grundlegenden Bundesgerichtsentscheid BGE $116 \mathrm{lb} 50 \mathrm{ff}$., bekannt unter dem Stichwort Deponie Chrüzlen, Egg/Oetwil.

${ }^{8}$ BGE $115 \mathrm{lb} 311 \mathrm{ff}$. (Starkstromleitung im Unterengadin); Bundesbeschluß betreffend das Konzept BAHN 2000 vom 19. Dezember 1986 (SR 742.100); Bundesbeschluß über den Bau der schweizerischen Eisenbahn-Alpentransversale (Alpentransit-Beschluß) vom 4. Okt. 1991, BBe 1991 III 1597.

${ }^{9}$ IP-BAU (DIGGELMANN/MURI/BARTL). Rechtliche Aspekte der Siedlungserneuerung, Bern 1991.
${ }^{10}$ Art. $25 \mathrm{ff}$. Bundesgesetz über die Raumplanung (RPG) vom 22. Juni 1979 (SR 700) sowie die kantonalen Planungs- und Baugesetze.

"Für die Dimensionierung der Bauzonen sind maßgebend: Art. 14 ff. RPG, sodann die Planungsgrundsätze gemäß Art. 3 RPG und die Aussagen des kantonalen Richtplans im Sinne von Art. $6 \mathrm{ff}$. RPG.

${ }^{12}$ Statt vieler Entscheide siehe insbesondere BGE 113 la 444 ff.; 114 la 368 ff.; 115 la 338 ff.; 116 la 341 ff.

${ }^{13}$ Art. 4, 25 und 33/34 RPG sowie weitere Bestimmungen des RPG und der Planungs- und Baugesetze.

${ }^{14}$ Art. $22^{\text {ter }}$ Abs. 3 BV und Art. 5 Abs. 2 RPG sowie eine reichhaltige Rechtsprechung des Bundesgerichts, vgl. beispielsweise BGE $116 \mathrm{lb} 14$ ff.; $116 \mathrm{lb} 384$ ff.; $117 \mathrm{lb} 6$ ff.; $113 \mathrm{lb} 322 \mathrm{ff}$.

${ }^{15}$ Art. 9 BG über den Umweltschutz (USG) vom 7. Oktober 1983 (SR 814.01); Verordnung über die Umweltverträglichkeitsprüfung (UVPV) vom 19. Oktober 1988 (SR 814.011).

${ }^{16}$ Art. 5 UVPV.

${ }^{17}$ BGE $116 \mathrm{lb} 50 \mathrm{ff}$.

${ }^{18}$ Art. 3 RPG.

${ }^{19}$ Zum Wesen und zur Funktion der Rechtsordnung siehe LENDI Martin, Rechtsordnung, a. a. O.

${ }^{20}$ Konvention zum Schutz der Menschenrechte und Grundfreiheiten (EMRK) vom 5. November 1950 (SR 0.101).

${ }^{21}$ Eine knappe, allgemein verständliche und gleichwohl präzise Darstellung des Rechts der EG enthält: EUROPÄISCHE DOKUMENTATION, Das Abc des Gemeinschaftsrechts, Brüssel 1990 (erhältlich beim Integrationsbüro in Bern). Im übrigen wird die Bundeskanzlei das für die Schweiz maßgebende Recht in einer besonderen Sammlung veröffentlichen, vorausgesetzt, die Schweiz tritt dem EWR bei.

${ }^{22}$ Wichtigste Grundlagen des EG-Rechts sind - aus der Sicht der Schweiz:

Vertrag zur Gründung der Europäischen Wirtschaftsgemeinschaft (EWG) vom 25. März 1957 mit seitherigen Änderungen,

Vertrag über die Europäische Union vom 7. Februar 1992 (vereinbart in Maastricht),

Abkommen über den europäischen Wirtschaftsraum (EWR) vom 2. Mai 1992 (vereinbart in Porto),

Abkommen zwischen der Europäischen Wirtschaftsgemeinschaft und der Schweizerischen Eidgenossenschaft über den Güterverkehr auf Straße und Schiene (Transitvertrag) vom 2. Mai 1992 (vereinbart in Porto, BBe 1992, III $1089 \mathrm{ff}$.).

Diese sind dargestellt in den in Ziff. 6 erwähnten bundesrätlichen Botschaften. Die Botschaft zum Transitvertrag - Botschaft zum Transitabkommen zwischen der Europäischen Gemeinschaft und der Schweiz, sowie zur trilateralen Vereinbarung zwischen der Bundesrepublik Deutschland, der Schweiz und Italien über den kombinierten Verkehr Schiene/ Straßevom 13. Mai 1992 - ist enthalten in BBe 1992 III 1057 ff. 